\title{
Covariant Formulation of the Transition Radiation Energy Spectrum of an Electron Beam at a Normal Angle of Incidence onto a Round Metallic Screen
}

\author{
Gian Luca Orlandi \\ Paul Scherrer Institut, 5232 Villigen PSI, CH.
}

\begin{abstract}
In the transition radiation emission from a $N$ electron bunch hitting at a normal angle of incidence a metallic screen, the transverse and the longitudinal spatial coordinates of the electron bunch play different roles in determining the $N$ single electron radiation field amplitudes and their relative phases in relation to the different physical constraints which an electromagnetic radiative mechanism by a charged beam must meet: i.e., temporal causality and covariance. The distribution of the $N$ electron longitudinal coordinates determines indeed the sequence of the $N$ electron collisions onto the metallic screen and, on the basis of the temporal causality principle, it also determines the distribution function of the relative emission phases of the $N$ single electron field amplitudes from the metallic surface. The distribution of the transverse coordinates of the $N$ electrons contributes as well to determine the relative phase distribution of the $N$ electron field amplitudes at the observation point - located on the longitudinal axis of the reference frame providing a further phase information that accounts for the transverse displacement of the $N$ electrons with respect to the beam axis. The distribution of the transverse coordinates of the $N$ electrons is a relativistic invariant under a Lorentz transformation with respect to the direction of motion of the beam and, consequently, it is expected to leave a covariant mark on the $N$ single electron amplitudes composing the radiation field. The covariant imprinting of the $N$ electron transverse density on the radiation field affects both the temporal coherent and incoherent parts of the transition radiation
\end{abstract}

Email address: gianluca.orlandi@psi.ch (Gian Luca Orlandi) 
energy spectrum. Such a dependence of the $N$ single electron radiation field amplitudes on the electron density in the transverse plane manifests itself as an increase - towards an asymptotic limit - of the radiated spectral energy with the decrease of the beam transverse size and as a spectral narrowing of the angular distribution of the radiation intensity with respect to the ideal case of a single electron hitting an infinite metallic screen. In the case of a round metallic screen with an arbitrary radius, the formal expression of the transition radiation energy spectrum will be derived and numerical results will be presented.

Keywords: Form Factor, Coherence, Fourier Transform, Collective Effects PACS: 41.60.-m, 41.75.-i, 42.25.Kb, 42.30.Kq

\section{Introduction}

A relativistic charge crossing a dielectric interface in a rectilinear and uniform motion can originate a highly directional and broad wavelength band radiation emission propagating backward and forward from the boundary surface within a small angle scaling down with the energy of the charge, the

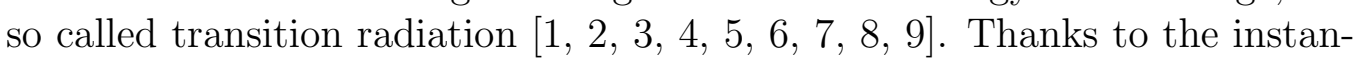
taneous, highly directional and charge-energy dependent features, transition radiation is a precious tool in the beam diagnostics of a particle accelerator. In the vacuum chamber of a particle accelerator the relativistic charged beam can be indeed intercepted by a movable thin metallic screen and the resulting light pulse can be imaged by a CCD-camera (Charge-Coupled-Device), for instance, to measure the transverse profile of the charged beam. Under the key-word OTR Beam Diagnostics (Optical Transition Radiation), the reader can find an enormous specialized literature dedicated to this topic. For most part of the practical detection conditions (from the extreme visible to the $\mathrm{THz}$ region), the metallic screen can be assimilated to an ideal conductor surface and the transition radiation emission can be easily schematized as the result of the dipolar oscillation of the conduction electrons of the metallic surface induced by the electromagnetic field of the incident relativistic charge. The dipolar oscillation induced in the double layer of charge by the relativistic charge explains indeed how an electromagnetic radiative mechanism, originated by a charge in a rectilinear and uniform motion, can also propagate in the backward direction. Such a model of the double layer of charge, describing the transition radiation emission as the result of the dipo- 
lar oscillation of the conduction electrons induced by the relativistic charge, is also enlightening about the kinematics of this radiative mechanism and about the common relativistic nature that it shares with other electromagnetic radiative mechanisms by relativistic charged beams. The kinematics of the transition radiative mechanism can be indeed schematized as the headon collision of two distributions of charge as observed in the reference frame of rest of one of the two colliding charged distributions. The backward and forward double conical transition radiation emission can be thus interpreted as the photon bremsstrahlung emission that two head-on colliding electron beams can originate. Taking into consideration that the transition radiation mechanism shares with other electromagnetic radiative mechanisms, such as the synchrotron or the bremsstrahlung radiation, the same kinematical and relativistic nature, it is thus reasonable to expect that, even at a very short wavelength, some spectral modifications of the radiation intensity due to transverse density of the beam should also affect the transition radiative mechanism by an electron beam in a similar way as, in other electromagnetic radiative mechanism by charged beams, the beam transverse size contributes to determine the so called Brilliance or Luminosity properties of the radiation source.

In the case of an electron beam at a normal angle of incidence onto a metallic screen with arbitrary size and shape, it can be demonstrated that a covariant and temporal causal formulation of the transition radiation energy spectrum of an electron beam necessarily implies, even at a very short wavelength, a dependence of the radiation spectral intensity on the distribution function of the particle density in the transverse plane [10]. In fact, in the transition radiation emission of an electron beam at a normal angle of incidence onto a metallic screen, the longitudinal and transverse coordinates of the electrons play different roles. The distribution function of the longitudinal coordinates of the $N$ electrons determines indeed the sequence of the particle collisions onto the metallic screen and, consequently, on the basis of the temporal causality principle, also the distribution function of the relative emission phases - from the metallic surface - of the $N$ single electron amplitudes composing the radiation field. The distribution function of the $N$ electron transverse coordinates contributes as well in determining the relative phase delay of the $N$ single electron field amplitudes at the observation point as a function of the transverse displacement of the $N$ electrons with respect to the beam axis where the radiation field is supposed to be observed. But, in addition, because of the relativistic invariance under a Lorentz trans- 
formation in the direction of motion of the beam, the $N$ electron transverse density manifests itself as a covariant feature of the radiation field whose observability can transform but not disappear under a Lorentz transformation. In the present work, the covariant and temporal causal formula of the transition radiation energy spectrum of a $N$ electron bunch, already derived in an implicit form in [10] in the most general case of a radiator surface with an arbitrary size and shape, will be here rendered into an explicit form in the particular case of a round radiator with an arbitrary radius. The so obtained formula of the transition radiation energy spectrum of a $N$ electron bunch at a normal angle of incidence onto a round metallic screen is demonstrated to be temporal causal and covariant [10] and to reproduce, as a limit, some results already well known in literature, such as: the Frank-Ginzburg formula of the single particle transition radiation energy spectrum in the ideal case of an infinite radiator or the single electron transition energy spectrum radiated by a round metallic screen with a finite radius. Finally, because of the covariant dependence of the radiation field on the transverse density of the $N$ electrons, it results that the distribution function of the $N$ electron transverse coordinates affects both the temporal coherent and incoherent parts of the transition radiation energy spectrum. Even at a very short wavelength, the effect of the $N$ electron transverse density on the spectral distribution of the radiation intensity manifests itself as an increase of the radiated energy with the decrease of the beam transverse size and a spectral narrowing of the angular distribution of the radiation intensity in comparison with the ideal case of an electron beam having a point-like transverse extension. In the following, in the case of a bunch of $N$ electrons hitting the metallic screen at a normal angle of incidence, details on the formula of the transition radiation energy spectrum and numerical results will be presented.

\section{Transition radiation energy spectrum of a $N$ electron bunch}

A bunch of $N$ electrons in a rectilinear and uniform motion along the $z$-axis of the laboratory reference frame is colliding, at a normal angle of incidence, onto a flat ideal conductor surface $S$ placed in the plane $z=0$. The $N$ electrons are supposed to fly in vacuum with a common velocity $\vec{w}=(0,0, w)$. All the electrons are supposed to hit the metallic screen at a normal angle of incidence. Effects of the angular divergence of the electron beam on the radiation energy spectrum are not considered in the present work. The radiator surface $S$ has a round shape with a finite radius $R$. The 
observation point of the radiation emission is on the $z$-axis at a distance $D$ from the screen much larger than the observed wavelength $\lambda$. The spatial coordinates of the $N$ electrons being $\left[\vec{\rho}_{0 j}=\left(x_{0 j}, y_{0 j}\right), z_{0 j}\right]$ with $j=1, \ldots, N$ at a given reference time $t=0$ when the center of mass of the charged distribution is crossing the boundary surface, the spectral component of the radiation field resulting from the collision of the $N$ relativistic electrons onto the metallic screen reads, see [10, 11, 12, 13, 14, 15, 16],

$$
E_{x, y}^{t r}(\vec{\kappa}, \omega)=\sum_{j=1}^{N} H_{x, y}\left(\vec{\kappa}, \omega, \vec{\rho}_{0 j}\right) e^{-i(\omega / w) z_{0 j}}
$$

where, under the far-field approximation [7, 9, 17], the single electron contribution to the radiation field can be calculated in the most general case of a radiator surface $S$ with an arbitrary shape and size (either infinite $S=\infty$ or finite $S<\infty)$ as follows [10, 11, 12, 13, 14, 15, 16]:

$$
H_{x, y}\left(\vec{\kappa}, \omega, \vec{\rho}_{0 j}\right)=\frac{i e k}{2 \pi^{2} D w} \int_{S} d \vec{\rho} \int d \vec{\tau} \frac{\tau_{x, y} e^{-i \vec{\tau} \cdot \vec{\rho}_{0 j}}}{\tau^{2}+\alpha^{2}} e^{i(\vec{\tau}-\vec{\kappa}) \cdot \vec{\rho}} .
$$

In previous Equation, $k=\omega / c=2 \pi / \lambda$ is the wave number, $\vec{\kappa}=\left(k_{x}, k_{y}\right)=$ $k \sin \theta(\cos \phi, \sin \phi)$ is the transverse component of the wave-vector, $\alpha=\frac{\omega}{w \gamma}$ ( $\gamma$ being the relativistic Lorentz factor) and the vector $\vec{\rho}=(x, y)$ represents the integration variable on the surface $S$ of the screen.

With reference to Eqs.(1).21), the transition radiation energy spectrum by a $N$ electron beam can be finally calculated as the flux of the Poynting vector, see also [10]:

$$
\frac{d^{2} I}{d \Omega d \omega}=\frac{c D^{2}}{4 \pi^{2}} \sum_{\mu=x, y}\left(\sum_{j=1}^{N}\left|H_{\mu, j}\right|^{2}+\sum_{j, l(j \neq l)=1}^{N} e^{-i(\omega / w)\left(z_{0 j}-z_{0 l}\right)} H_{\mu, j} H_{\mu, l}^{*}\right),
$$

where $H_{\mu, j}=H_{x, y}\left(\vec{\kappa}, \omega, \vec{\rho}_{0 j}\right)$ with $\mu=x, y$, as defined in Eq.(2) .

The formal expression of the radiation field and of the radiation energy spectrum of the $N$ electron bunch, as represented in Eqs.(1/2) and Eq.(3) in the most general case of a flat radiator surface $S$ with an arbitrary size and shape, meets the constraints of the temporal causality and of the covariance. The structure of the emission phases from the screen of the $N$ single electron field amplitudes composing the radiation field - see Eqs.(1),2) - is 
indeed causally related to the temporal sequence of the $N$ particle collision onto the metallic screen. Furthermore, with reference to [10], covariant or covariance consistent are all the formal steps which, from the expression of the electromagnetic field of the $N$ electron bunch in the laboratory reference frame, leads to the radiation field - see Eqs.(1),2) - and to the radiation energy spectrum, see $\mathrm{Eq}(3)$. For further details about the covariant and the temporal causal formulation of the transition radiation emission from a $N$ electron bunch - as described in Eqs.(1, 2, 3) - the reader is addressed to [10].

For the sake of ease, in order to proceed with the formal explicit derivation of the transition radiation energy spectrum in the case of a round radiator with a radius $R$, a polar angle representation is considered for the following vectors

$$
\left\{\begin{array}{l}
\vec{\tau}=\tau(\cos \eta, \sin \eta) \\
\vec{\xi}_{j}=\left(\vec{\rho}-\vec{\rho}_{0 j}\right)=\xi_{j}\left(\cos \psi_{j}, \sin \psi_{j}\right)
\end{array}\right.
$$

where $\xi_{j}=\sqrt{\rho^{2}+\rho_{0 j}^{2}-2 \vec{\rho} \cdot \vec{\rho}_{0 j}}(j=1, . ., N), \vec{\rho}_{0 j}=\left(x_{0 j}, y_{0 j}\right)$ and $\vec{\rho}=(x, y)$ with $0<\rho<R$ being the vectors of the $N$ electron transverse coordinates and the vector of the screen coordinates, respectively. Taking into account the following integral representation of the Bessel function of the first kind [18] (see also Eq.(17) in [19]):

$$
\int_{0}^{2 \pi} d \psi\left(\begin{array}{c}
\cos \psi \\
\sin \psi
\end{array}\right) e^{i a b \cos (\psi-\phi)}=2 \pi i\left(\begin{array}{c}
\cos \phi \\
\sin \phi
\end{array}\right) J_{1}(a b)
$$

in the limit of a round metallic surface with a radius $R$ much longer than the average transverse size of the beam $\left(R \gg<\rho_{0 j}>, j=1, . ., N\right)$ the integral that, in Eq.(2), determine the single electron field amplitude can be explicitly calculated:

$$
\begin{aligned}
& \int_{S} d \vec{\rho} \int d \vec{\tau} \frac{\tau_{x, y} e^{-i \vec{\tau} \cdot \vec{\rho}_{0 j}}}{\tau^{2}+\alpha^{2}} e^{i(\vec{\tau}-\vec{\kappa}) \cdot \vec{\rho}}= \\
& =\int_{S} d\left(\vec{\rho}-\vec{\rho}_{0 j}\right) e^{-i \vec{\kappa} \cdot\left(\vec{\rho}-\vec{\rho}_{0 j}+\vec{\rho}_{0 j}\right)} \int d \vec{\tau} \frac{\tau_{x, y} e^{i \vec{\tau} \cdot\left(\vec{\rho}-\vec{\rho}_{0 j}\right)}}{\tau^{2}+\alpha^{2}}= \\
& =e^{-i \vec{\kappa} \cdot \vec{\rho}_{0 j}} \int d \vec{\xi}_{j} e^{-i \vec{\kappa} \cdot \vec{\xi}_{j}} \int d \vec{\tau} \frac{\tau_{x, y} e^{i \vec{\tau} \cdot \vec{\xi}_{j}}}{\tau^{2}+\alpha^{2}}= \\
& =e^{-i \vec{\kappa} \cdot \vec{\rho}_{0 j}} \int d \vec{\xi}_{j} e^{-i \vec{\kappa} \cdot \vec{\xi}_{j}} \int \frac{d \eta d \tau \tau^{2}}{\tau^{2}+\alpha^{2}}\left(\begin{array}{c}
\cos \eta \\
\sin \eta
\end{array}\right) e^{i \tau \xi_{j} \cos \left(\eta-\psi_{j}\right)}=
\end{aligned}
$$




$$
\begin{aligned}
& =(2 \pi i) e^{-i \vec{\kappa} \cdot \vec{\rho}_{0 j}} \int d \xi_{j} \xi_{j} d \psi_{j}\left(\begin{array}{c}
\cos \psi_{j} \\
\sin \psi_{j}
\end{array}\right) e^{-i \kappa \xi_{j} \cos \left(\psi_{j}-\phi\right)} \int_{0}^{\infty} \frac{d \tau \tau^{2} J_{1}\left(\tau \xi_{j}\right)}{\tau^{2}+\alpha^{2}}= \\
& =(2 \pi)^{2} \alpha\left(\begin{array}{c}
\cos \phi \\
\sin \phi
\end{array}\right) e^{-i \vec{\kappa} \cdot \vec{\rho}_{0 j}} \int_{\rho_{0 j}}^{R+\rho_{0 j}} d \xi_{j} \xi_{j} K_{1}\left(\alpha \xi_{j}\right) J_{1}\left(\kappa \xi_{j}\right)= \\
& =(2 \pi)^{2}\left(\begin{array}{c}
\cos \phi \\
\sin \phi
\end{array}\right) \frac{\kappa e^{-i \vec{\kappa} \cdot \vec{\rho}_{0 j}}}{\kappa^{2}+\alpha^{2}}\left[\rho_{0 j} \Phi\left(\kappa, \alpha, \rho_{0 j}\right)-\left(R+\rho_{0 j}\right) \Phi\left(\kappa, \alpha, R+\rho_{0 j}\right)\right]
\end{aligned}
$$

with

$$
\Phi\left(\kappa, \alpha, \rho_{0 j}\right)=\alpha J_{0}\left(\kappa \rho_{0 j}\right) K_{1}\left(\alpha \rho_{0 j}\right)+\frac{\alpha^{2}}{\kappa} J_{1}\left(\kappa \rho_{0 j}\right) K_{0}\left(\alpha \rho_{0 j}\right)
$$

and

$$
\Phi\left(\kappa, \alpha, R+\rho_{0 j}\right)=\alpha J_{0}\left[\kappa\left(R+\rho_{0 j}\right)\right] K_{1}\left[\alpha\left(R+\rho_{0 j}\right)\right]+\frac{\alpha^{2}}{\kappa} J_{1}\left[\kappa\left(R+\rho_{0 j}\right)\right] K_{0}\left[\alpha\left(R+\rho_{0 j}\right)\right]
$$

where Eqs.(56].77) follows from Eq.(44) and from formulas 6.566(2), 6.521(4), $8.473(1)$ and $8.486(17)$ in [18].

In conclusion, with reference to Eqs.(5],6]7), the harmonic component of the radiation field produced by a $N$ electron bunch hitting a round radiator surface with a finite radius $R$ reads, see Eqs.(1]2),

$$
\begin{aligned}
E_{x, y}^{t r}(\vec{\kappa}, \omega) & =\sum_{j=1}^{N} H_{x, y}\left(\vec{\kappa}, \omega, \vec{\rho}_{0 j}\right) e^{-i(\omega / w) z_{0 j}}=\sum_{j=1}^{N} \frac{2 i e k}{D w} \frac{\kappa}{\kappa^{2}+\alpha^{2}} e^{-i\left[(\omega / w) z_{0 j}+\vec{\kappa} \cdot \vec{\rho}_{0 j}\right]} \times \\
& \times\left(\begin{array}{c}
\cos \phi \\
\sin \phi
\end{array}\right)\left[\rho_{0 j} \Phi\left(\kappa, \alpha, \rho_{0 j}\right)-\left(R+\rho_{0 j}\right) \Phi\left(\kappa, \alpha, R+\rho_{0 j}\right)\right]
\end{aligned}
$$

where $\vec{\kappa}=\left(\kappa_{x}, \kappa_{y}\right)=k \sin \theta(\cos \phi, \sin \phi)$ is the transverse component of the wave-vector $(k=2 \pi / \lambda)$. In some special and relevant cases, the formula of the transition radiation field given in Eqs.(6],7],8) reproduces as a limit some results already well known in literature.

Case1. Single electron $(j=1)$ and $\left(\rho_{0 j}=0, R=\infty\right)$ : i.e., a single electron moving on the $z$-axis $(x=y=0)$, where the observation point is also located, in collision onto a radiator having an infinite surface. In the limit $R \rightarrow \infty$ and $\rho_{0 j} \rightarrow 0$, the functions defined in Eqs.(6],7]) tend to the following limit values:

$$
\left\{\begin{array}{l}
\left(R+\rho_{0 j}\right) \Phi\left(\kappa, \alpha, R+\rho_{0 j}\right) \rightarrow 0 \\
\rho_{0 j} \Phi\left(\kappa, \alpha, \rho_{0 j}\right) \rightarrow 1
\end{array}\right.
$$


Taking into account the limits above, the following expression of the single electron radiation field, see Eqs.(6],7,8), follows

$$
E_{x, y}^{t r}(\vec{\kappa}, \omega)=\frac{2 i e k}{D w} \frac{\kappa}{\kappa^{2}+\alpha^{2}}\left(\begin{array}{c}
\cos \phi \\
\sin \phi
\end{array}\right)
$$

where $\kappa=k \sin \theta$. Finally, from the Equation above and Eq.(3), the well known result of the Frank-Ginzburg formula of the transition radiation energy spectrum in the ideal case of a single electron hitting an infinite ideal conductor surface can be obtained [1, 2, 3, 4, 5, 6, 7, 8]:

$$
\frac{d^{2} I_{e}}{d \Omega d \omega}=\frac{(e \beta)^{2}}{\pi^{2} c} \frac{\sin ^{2} \theta}{\left(1-\beta^{2} \cos ^{2} \theta\right)^{2}} .
$$

Case 2. Single electron $(j=1)$ and $\left(\rho_{0 j}=0, R<\infty\right)$ : similar experimental situation as in previous case but finite radius of the radiator. With reference to the above reported limit of the expression in Eq.(6) for $\rho_{0 j} \rightarrow 0$, the radiation field reads, see Eqs.(1,2,5,6,7),

$$
E_{x, y}^{t r}(\vec{\kappa}, \omega)=\frac{2 i e k}{D w} \frac{\kappa}{\kappa^{2}+\alpha^{2}}\left(\begin{array}{c}
\cos \phi \\
\sin \phi
\end{array}\right)\left[1-\alpha R J_{0}(\kappa R) K_{1}(\alpha R)-\frac{\alpha^{2} R}{\kappa} J_{1}(\kappa R) K_{0}(\alpha R)\right]
$$

which, in the far-field approximation, represents the radiation field of a single electron hitting a finite round metallic screen. In the case of a single electron hitting a round metallic screen with a finite radius, as a function of the transverse extension $(\gamma \lambda / 2 \pi)$ of the single electron virtual quanta field compared to the finite radius $R$ of the radiator, the transition radiation energy spectrum experiences a low frequency diffractive cut-off. This problem was already tackled in several works [20, 21, 22, 23, 24, 25]. Compare Eq.(11) in the present work with Eqs. $(8,9)$ in [25], for instance.

Finally, from Eqs.(38), the explicit expression of the transition radiation energy spectrum of a $N$ electron bunch colliding onto a round ideal conductor surface with a finite radius $R$ can be obtained

$$
\frac{d^{2} I}{d \Omega d \omega}=\frac{d^{2} I_{e}}{d \Omega d \omega}\left(\sum_{j=1}^{N}\left|A_{j}\right|^{2}+\sum_{j, l(j \neq l)=1}^{N} A_{j} A_{l}^{*} e^{-i\left[(\omega / w)\left(z_{0 j}-z_{0 l}\right)+\vec{\kappa} \cdot\left(\vec{\rho}_{0 j}-\vec{\rho}_{0 l}\right)\right]}\right)
$$

where $\frac{d^{2} I_{e}}{d \Omega d \omega}$ is the single electron radiation energy spectrum already defined in Eq.(10) and

$$
A_{j}=\rho_{0 j} \Phi\left(\kappa, \alpha, \rho_{0 j}\right)-\left(R+\rho_{0 j}\right) \Phi\left(\kappa, \alpha, R+\rho_{0 j}\right) \simeq \rho_{0 j} \Phi\left(\kappa, \alpha, \rho_{0 j}\right),
$$


the approximation in Eq.(13) being valid for most of the experimental situations where diffractive modifications of the spectral distribution of the radiated energy due to the finite size of the screen can be neglected.

In the formula of the transition radiation energy spectrum of a $N$ electron beam, as explicitly derived in Eqs.(12,13) in the case of a normal collision onto a round metallic screen with a finite radius $R$, the double role played by the transverse coordinates of the $N$ electrons appears evident. On the one hand, as a function of the displacement of the electrons with respect to the beam axis where the radiation field is supposed to be observed, they contribute to determine the relative phase delay of the $N$ electron field amplitudes at the observation point as the presence of the well known threedimensional phase factor in the temporal coherent part of the formula given in Eq.(12) clearly indicates. On the other hand, because of the invariance of the transverse density of the $N$ electrons under a Lorentz transformation in the direction of motion of the beam, the $N$ single electron radiation field amplitudes show a covariant dependence on the transverse density of the $N$ electron beam. As a result, see Eqs.(12,13), both the temporal coherent and the temporal incoherent components of the transition radiation energy spectrum of a $N$ electron beam differ from the ideal scenario of a single electron hitting an infinite metallic screen. It results indeed that, even at a very short wavelength, the radiation spectral intensity increases with the decrease of the beam transverse size towards an asymptotic limit as well as the spectral angular distribution of the radiation experiences a broadening towards the asymptotic limit given by the well known ideal case of a single electron hitting an infinite metallic surface.

In Figs.(1,2,3,4), results of the numerical simulation of the temporal incoherent part of the transition radiation spectrum produced by a gaussian electron bunch with a transverse size much shorter than the finite radius of the radiator are shown, see Eqs.(1213). In Fig.(11), for a beam energy of $500 \mathrm{MeV}$ and a bunch of $N=10^{5}$ electrons, the angular distribution of the transition radiation intensity in the visible (Green, $\lambda=530 \mathrm{~nm}$ ) for different values of the beam standard deviation $\sigma=10,50,100 \mu \mathrm{m}$ is calculated and compared with the ideal result obtainable from a bunch with the same charge but radiating according to the formula given in Eq.(10). In Figs.(2,3]4), for different beam energies 500,750,1000 $\mathrm{MeV}$ and a constant beam charge of $N=10^{5}$ electrons, the angular distributions of the transition radiation intensity radiated at different wavelengths in the visible (Red, Green, Blue $\lambda=680,530,400 \mathrm{~nm}$ ) by a beam with standard deviation $\sigma=50 \mu \mathrm{m}$ are 
compared with the analogous result obtained in the ideal case from the formula in Eq.(10).

\section{Conclusions}

Temporal causality and covariance constrain the role that the distributions of the longitudinal and transverse coordinates of an electron bunch plays in determining the physical features of an electromagnetic radiative mechanism, in particular, the transition radiation mechanism. In the case of a $N$ electron bunch hitting at a normal angle of incidence a metallic screen, the distribution of the electron longitudinal coordinates defines the causal correlation occurring between the temporal sequence of the electron collisions onto the metallic screen and the structure of the relative emission phases of the $N$ single electron radiation field amplitudes propagating from the metallic screen to the observation point, located on the beam longitudinal axis. The distribution of the transverse coordinates of the $N$ electrons contributes as well to determine the relative phase delay of the $N$ single electron radiation field amplitudes at the observation point as a function of the $N$ electron displacements with respect to the beam axis. The transverse density of the $N$ electrons, being an invariant under a Lorentz transformation with respect to the direction of motion of the beam, constitutes for the radiation field a covariant feature that affects both the spectral intensity and the angular distributions of the transition radiation under observation conditions of both temporal incoherence and temporal coherence. Compared to the reference asymptotic results provided by the well known ideal formalism of a single electron hitting an infinite metallic screen, an increase of the radiated spectral intensity with the decrease of the beam transverse size and a broadening of the spectral angular distribution constitute the relevant phenomenological aspects of the covariant beam-transverse-size effects on the transition radiation energy spectrum originated by an electron beam hitting a round metallic screen at a normal angle of incidence.

\section{References}

[1] V. L. Ginzburg and I. M. Frank, Soviet Phys. JETP 16 (1946) 15.

[2] G.M. Garibian, Sov. Phys. JETP, 6 (33), 6 (1958) 1079-1085.

[3] G.M. Garibyan, Sov. Phys. JETP, 37 (10), 2 (1960) 372-376. 
[4] I.M. Frank, Sov. Phys. Usp., 4, 5, (1962) 740-746.

[5] F.G. Bass, V. M. Yakovenko, Sov. Phys. Usp., 8, 3 (1965) 420-444.

[6] I.M. Frank, Sov. Phys. Usp., 8, 5 (1966) 729-741.

[7] M.L. Ter-Mikaelian, High-Energy Electromagnetic Processes in Condensed Media, Wiley, New York (1972).

[8] V.L. Ginzburg, V.N. Tsytovich, Transition Radiation and Transition Scattering, Adam Hilger, Bristol (1990).

[9] J.D. Jackson, Classical Electrodynamics, Wiley, New York (1975).

[10] G.L. Orlandi, On the covariance of the charge form factor in the transition radiation energy spectrum of a beam at normal incidence onto a metallic screen, Paul Scherrer Institut Preprint PSI-PR-12-03.

[11] G.L. Orlandi, Opt. Commun. 211 (2002) 109-119.

[12] G.L. Orlandi, Opt. Commun. 267 (2006) 322-334.

[13] G.L. Orlandi, Proc. FEL 2005, Stanford University (2005), JACoW / eConf C0508213, 576-579.

[14] G.L. Orlandi, Proc. EPAC 08, Genoa, Italy, (2008) 1221-1223.

[15] G.L. Orlandi, B. Beutner, R. Ischebeck, V. Schlott, B. Steffen, Proc. FEL 2009, Liverpool, UK, 153 (2009).

[16] G.L. Orlandi, R. Ischebeck, V. Schlott, B. Steffen, Proc. FEL 2010, Malmö, Sweden, (2010).

[17] M. Born, E. Wolf, Principles of Optics, Pergamon Press, Oxford (1965).

[18] I.S. Gradshteyn and I.M. Ryzhik, Table of Integrals, Series, and Products, $5^{\text {th }}$ Ed. (1998) Academic Press.

[19] M. Castellano and V.A. Verzilov, Phys. Rev. ST-AB 1, 062801 (1998).

[20] N.F. Shul'ga, S.N. Dobrovol'sky, JETP Lett., Vol. 65, No. 8, (1997) 611-614. 
281

[21] A.P. Potylitsyn, Nucl. Instr. and Meth. in Phys. Res. B 145 (1998) $169-179$

[22] M. Castellano, A. Cianchi, G. Orlandi, V.A. Verzilov, Nucl. Instr. and Meth. in Phys. Res. A 435 (1999) 297-307.

[23] S. Casalbuoni, B. Schmidt and P. Schmüser, TESLA Report 2005-15 (2005).

[24] D. Sütterlin, D. Erni, M. Dehler, H. Jäckel, H. Sigg, V. Schlott, Nucl. Instr. and Meth. in Phys. Res. B 264 (2007) 361-370.

[25] S. Casalbuoni, B. Schmidt,P. Schmüser, V. Arsov, S. Wesch, Phys. Rev. ST-AB 12, 030705 (2009). 


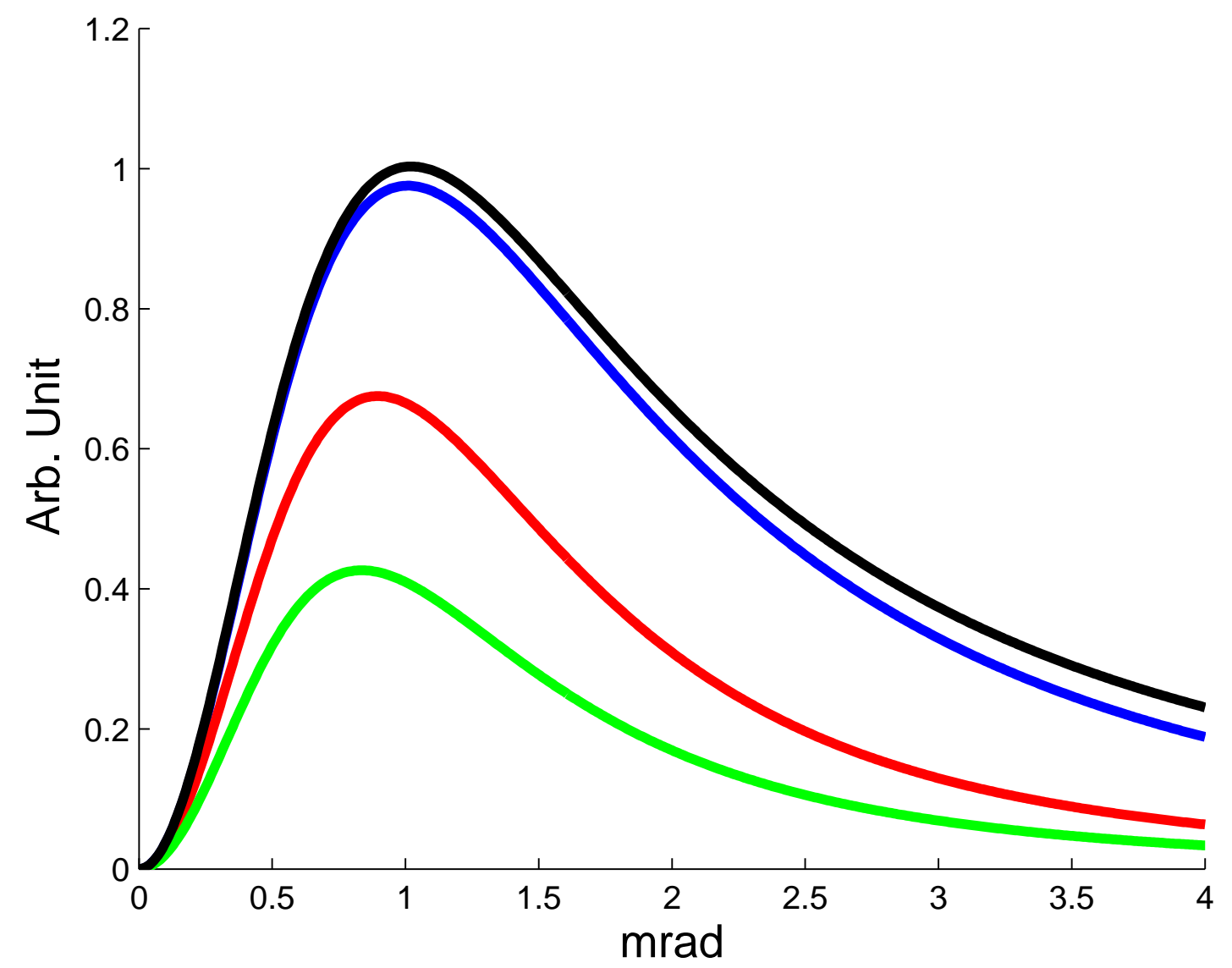

Figure 1: Angular distribution of the transition radiation emitted at a wavelength $\lambda=530$ $\mathrm{nm}$ by a gaussian bunch of $N=10^{5}$ electrons and energy $500 \mathrm{MeV}$ with standard deviation $\sigma=10 \mu m$ (Blue curve), $\sigma=50 \mu m$ (Red curve) and $\sigma=100 \mu m$ (Green curve). The Blue, Red and Green curves are calculated via Eqs.112]13) and compared with the analogous result (Black curve) that can be calculated in the same conditions of beam energy and charge via Eq.(10). 


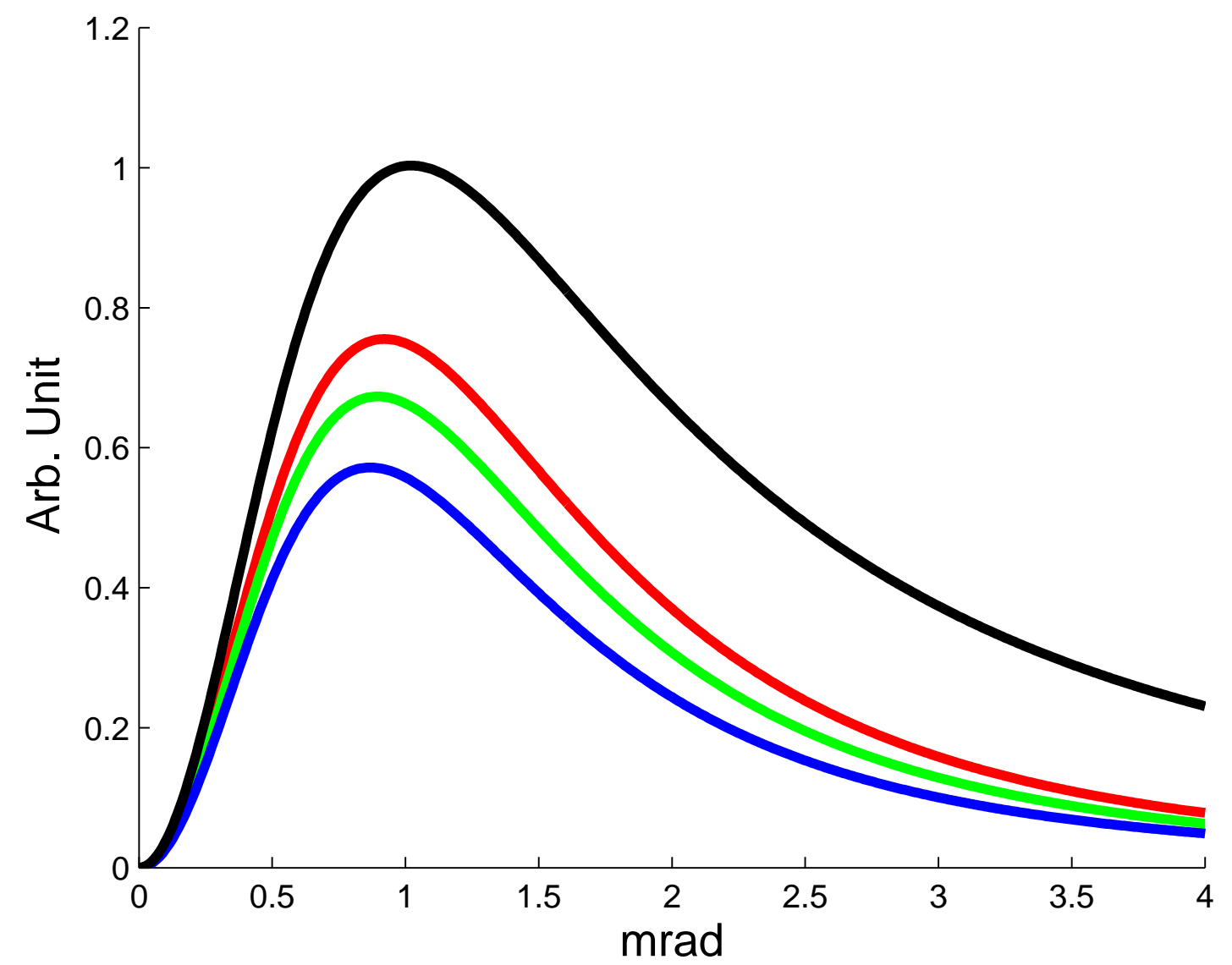

Figure 2: Beam energy $500 \mathrm{MeV}$. Angular distribution of the transition radiation emitted at a wavelength $\lambda=680 \mathrm{~nm}$ (Red curve), $\lambda=530 \mathrm{~nm}$ (Green curve), $\lambda=400 \mathrm{~nm}$ (Blue curve) by a gaussian bunch of $N=10^{5}$ electrons with standard deviation $\sigma=50 \mu \mathrm{m}$. The Blue, Red and Green curves are calculated via Eqs.(12/13) and compared with the analogous result (Black curve) that can be calculated in the same conditions of beam energy and charge via Eq.(10). 


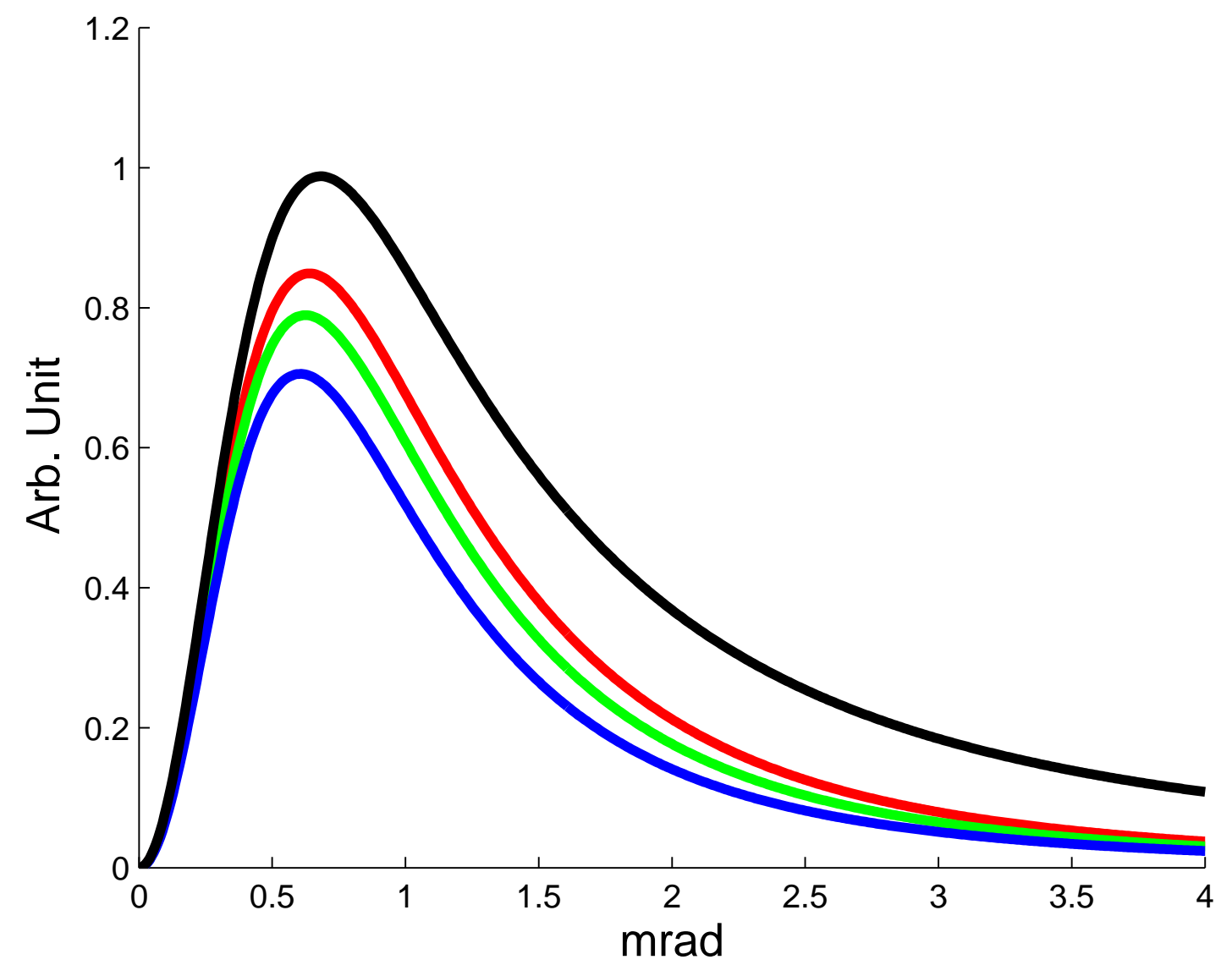

Figure 3: Beam energy $750 \mathrm{MeV}$. Angular distribution of the transition radiation emitted at a wavelength $\lambda=680 \mathrm{~nm}$ (Red curve), $\lambda=530 \mathrm{~nm}$ (Green curve), $\lambda=400 \mathrm{~nm}$ (Blue curve) by a gaussian bunch of $N=10^{5}$ electrons with standard deviation $\sigma=50 \mu \mathrm{m}$. The Blue, Red and Green curves are calculated via Eqs.(12]13) and compared with the analogous result (Black curve) that can be calculated in the same conditions of beam energy and charge via Eq.(10). 


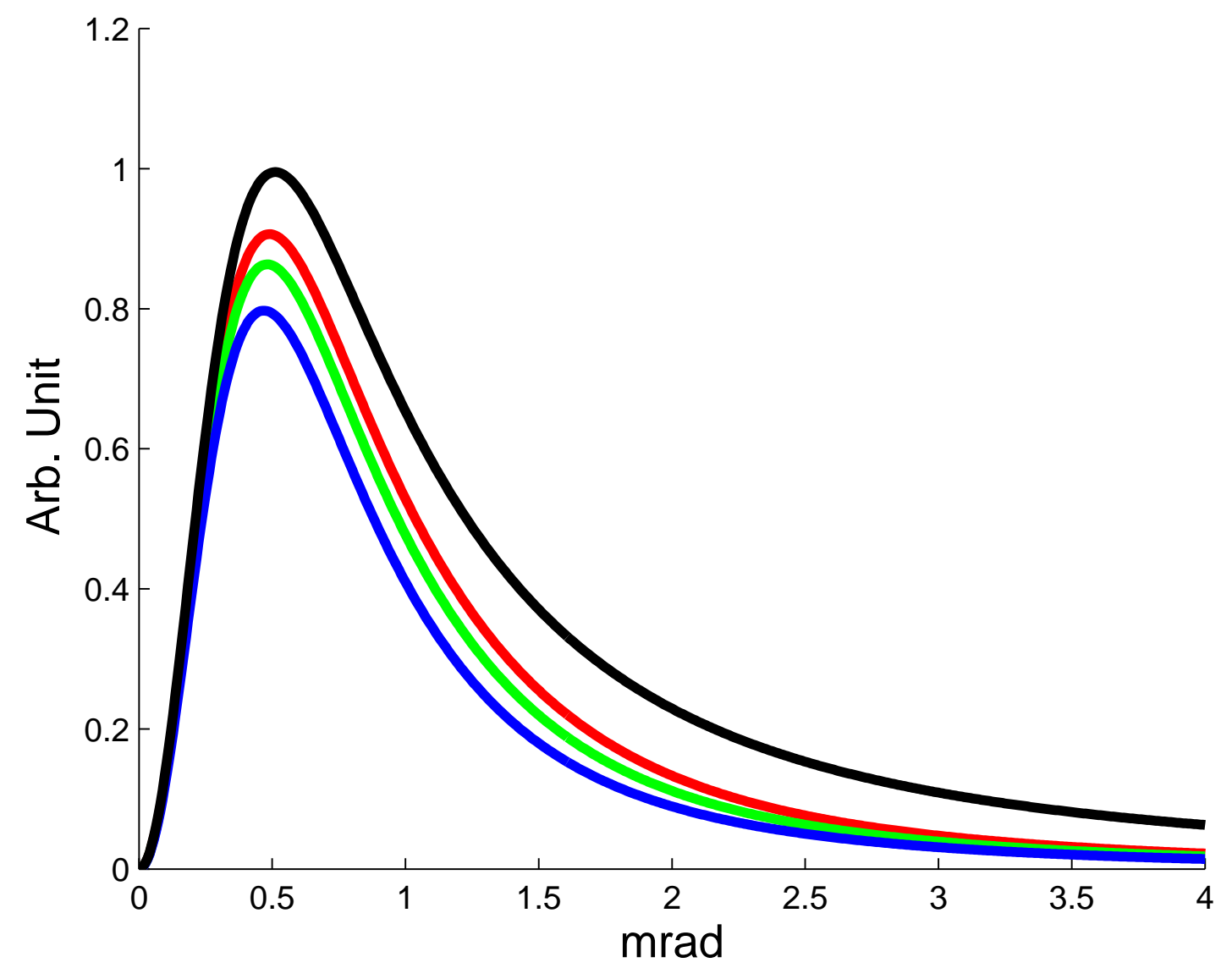

Figure 4: Beam energy $1000 \mathrm{MeV}$. Angular distribution of the transition radiation emitted at a wavelength $\lambda=680 \mathrm{~nm}$ (Red curve), $\lambda=530 \mathrm{~nm}$ (Green curve), $\lambda=400 \mathrm{~nm}$ (Blue curve) by a gaussian bunch of $N=10^{5}$ electrons with standard deviation $\sigma=50 \mu \mathrm{m}$. The Blue, Red and Green curves are calculated via Eqs.(12]13) and compared with the analogous result (Black curve) that can be calculated in the same conditions of beam energy and charge via Eq.(10). 